\title{
GAIA Level 1 Antepartum Stillbirth
}

National Cancer Institute

\section{Source}

National Cancer Institute. GAIA Level 1 Antepartum Stillbirth. NCI Thesaurus. Code C128018.

GAIA Level 1 Antepartum Stillbirth is defined by four criteria: first, one or more of the following three requirements must be met: a) Prenatal ultrasound examination (e.g. basic ultrasound or special ultrasound with Doppler) documenting an absence of fetal cardiac activity or movement before the onset of labor; b) Radiology findings consistent with intrauterine fetal death; c) Delivery of an infant with no of signs of life at birth, including no spontaneous movements, no umbilical cord pulse, no heartbeat, no respirations, and Apgar scores of 0 at both 1 and 5 minutes. The determination of the absence of signs of life is made by physical examination after delivery, with or without electronic monitoring of heart rate, respiratory rate, and pulse oximetry; second, the following three criteria must be met: a) Maternal report of the absence of fetal movement for 24 hours or more; b) Maternal physical examination confirming the absence of fetal movement; c) Auscultation for fetal heart tones using electronic devices (e.g. handheld Doppler or fetal heart rate monitor) OR non-electronic devices (e.g. stethoscope, fetoscope, or Pinard horn) that documents the absence of a fetal heartbeat; third, one of the following two requirements must be met: a) Attended delivery, followed by a post-delivery physical examination of the fetus by an obstetrician, a neonatologist, a pediatrician, a maternalfetal medicine specialist, or a pathologist that is consistent with antepartum death. In a setting where access to a specialist is not feasible, diagnosis by a health care provider trained or experienced to make the diagnosis is acceptable (e.g. general practice physician, mid-wife, nurse practitioner, a physician's assistant, or other qualified trained practitioner); OR b) fetal/placental pathology report that is consistent with antepartum death; fourth, gestational age within the pre-defined range for selected stillbirth definition, as assessed by maternal and/or fetal parameters (level 1 in GA assessment algorithm). 